\title{
Macrophage-Biomimetic Nanoparticles Ameliorate Ulcerative Colitis through Reducing Inflammatory Factors Expression
}

\author{
Zhengshuo Li $i^{\text {a, b, c }}$ Xiaoyue Zhang ${ }^{\text {b, c }}$ Can Liu ${ }^{\text {b, c }}$ Qiu Peng ${ }^{b, c}$ Yangge Wu ${ }^{\text {b, c }}$ \\ Yuqing Wen $^{b, c}$ Run Zheng ${ }^{b, c}$ Qun Yan ${ }^{d}$ Jian Ma ${ }^{a, b}, c$ \\ aHunan Key Laboratory of Cancer Metabolism, Hunan Cancer Hospital and the Affiliated Cancer Hospital of Xiangya \\ School of Medicine, Central South University, Changsha, China; ${ }^{b}$ Cancer Research Institute and School of Basic \\ Medical Science, Central South University, Changsha, China; `Key Laboratory of Carcinogenesis and Cancer Invasion \\ of the Chinese Ministry of Education, NHC Key Laboratory of Carcinogenesis, Hunan Key Laboratory of Nonresolving \\ Inflammation and Cancer, Changsha, China; 'Department of Clinical Laboratory, Hematology, Xiangya Hospital, \\ Central South University, Changsha, China
}

\section{Keywords}

Ulcerative colitis · S100A9 inhibitor · Biomimetic

nanoparticles $\cdot$ Drug delivery system

\begin{abstract}
Background and Aims: Inflammatory mediator S100A9 is dramatically elevated in ulcerative colitis and correlates with disease severity. S100A9 is a potential molecule to target for the treatment of colitis, but to date, there is no effective targeting method. The aim of this study was to develop a safe and effective nano-delivery system targeting S100A9 and to evaluate its therapeutic efficacy in ulcerative colitis mouse model. Methods: We designed an oral nano-delivery system using poly (lactic acid-glycolic acid) (PLGA)-loaded S100A9 inhibitor tasquinimod to synthesize PLGA-TAS nanoparticles. TLR4-overexpressing macrophage membranes (MMs) were used to wrap the nanoparticles to make MM-PLGA-TAS, which allowed the nanoparticles to acquire the ability to specifically enrich the colitis region. Results: MM-PLGA-TAS was endocytosed by inflammatory phenotype RAW264.7 cells in vitro and can efficiently enrich in inflamed mouse
\end{abstract}

karger@karger.com www.karger.com/jin

Karger!"

BOPEN ACCESS
(C) 2021 The Author(s)

Published by S. Karger AG, Basel

This is an Open Access article licensed under the Creative Common Attribution-NonCommercial-4.0 International License (CC BY-NC) (http://www.karger.com/Services/OpenAccessLicense), applicable to the online version of the article only. Usage and distribution for commercial purposes requires written permission. colitis tissue in vivo. A chemically induced ulcerative colitis mouse model was used to evaluate the therapeutic effect of oral MM-PLGA-TAS. MM-PLGA-TAS significantly alleviated the symptoms of ulcerative colitis, and mechanically, MMPLGA-TAS achieved immunomodulatory and suppressive effects by reducing S100a9 and other cytokines in the colitis region. Conclusion: We describe a convenient, orally targeted colitis drug delivery system that cures the disease in ulcerative colitis mice. This system substantially increases drug accumulation in inflamed colonic tissue, reduces the risk of systemic exposure, and is a promising therapeutic approach against ulcerative colitis.

(c) 2021 The Author(s) Published by S. Karger AG, Basel

\section{Introduction}

Inflammatory bowel disease (IBD) mainly includes ulcerative colitis and Crohn's disease. Ulcerative colitis is a chronic nonspecific inflammatory disease that affects the colorectal mucosa, with a long period of onset and
Correspondence to:

Jian Ma, majian@csu.edu.cn 
easy recurrence [1-3]. The pharmacological treatment used to treat patients with ulcerative colitis is mainly based on the daily administration of glucocorticoids and the use of immunosuppressive agents $[4,5]$. Biological inhibitors targeting the overactive adaptive immune system, such as infliximab, an anti-TNFa monoclonal antibody, are a new avenue for targeted treatment of ulcerative colitis [6, 7]. However, the use of the required doses of corticosteroids, immunosuppressants, and anti-TNFa monoclonal antibody drugs cannot be administered for long periods due to the presence of systemic adverse side effects $[7,8]$. This leaves many limitations to conventional drugs in the fight against ulcerative colitis and requires the development of novel targeted drug delivery systems to mitigate the occurrence of systemic adverse effects.

Nanotechnology-based colon-targeted drug system has recently gained attention because of its potential to accumulate in inflamed colonic tissue. Due to the increased permeability of inflamed tissues [9], nanoparticle-based delivery systems can induce the specific accumulation of loaded drugs in inflamed tissues. Recently, bionic drug delivery systems, especially cell membraneencapsulated nanoparticles have demonstrated better therapeutic efficacy against inflammatory diseases because of their higher biocompatibility and ability to specifically target inflamed tissues and organs. Macrophage membrane (MM)-encapsulated nanoparticles not only specifically accumulate at the site of inflammation due to their inflammatory homing effect $[10,11]$, but also absorb lipopolysaccharide (LPS) and inflammatory cytokines to modulate and suppress local inflammation. Thus, cell membrane-encapsulated nanobionic drug delivery systems may be a solution to the current ulcerative colitis drug delivery dilemma.

S100A9 is a damage-associated molecular model protein released mainly by neutrophils and monocytes and belongs to the $\mathrm{S} 100$ family of $\mathrm{Ca}^{+}$-binding proteins, which often plays a decisive role in the development of nonresolving inflammation together with S100A8 [12]. In our previous work, we found that S100a9 protein was the most significantly upregulated protein in a mouse model of dextran sulfate sodium (DSS)-induced ulcerative colitis [13]. Treatment with anti-S100a9 antibody resulted in a significant decrease in the frequency of neutrophils, macrophages, and dendritic cells in the colon tissues of ulcerative colitis mice, a downregulation of the expression of inflammatory factors, such as S100a9, Tnfa, IL1 $\beta$, IL6, IL17a, Ifn $\gamma$, IL12a, IL23a, and a significant reduction in intestinal inflammatory symptoms in mice [14]. We previously reported that S100A8/9 significantly increase the PDL1 expression levels [15]. It is suggested that S100a9 is a promising therapeutic target for the treatment of IBD-related inflammatory symptoms. Currently, there is no established monoclonal antibody targeting regimen for S100a9 molecule for clinical use, and further development of effective strategies to target S100a9 in the inflammatory region is needed.

In this study, we developed nanocarriers loaded with the S100A9 inhibitor tasquinimod (TAS) to enhance the inflammatory targeting of nanoparticles. Poly (lactic acid-glycolic acid) (PLGA) has excellent biocompatibility and adequate biodegradability and is therefore widely used as a matrix for nanocarriers [16]. First, PLGA loaded with TAS was prepared by the solvent evaporation method. Subsequently, these nanoparticles (PGLA-TAS) and TLR4-overexpressing MMs were fully mixed to finally obtain the synthetic MM-PLGA-TAS nanocarriers. High expression of S100a9 inflammatory regions can recruit macrophages [13], and its receptor molecules on MMs can aid the enrichment of nanoparticles in inflammatory regions, while TLR4, an S100a9specific receptor, overexpressed on cell membranes, can enhance this process. This new drug and targeting system may be a promising therapeutic approach for the treatment of IBD.

\section{Materials and Methods}

\section{PLGA-TAS/DIR Preparation}

The S100A9 inhibitor TAS (10 mg; MedChemExpress) and PLGA (120 mg; Sigma-Aldrich) were dissolved in $5 \mathrm{~mL}$ dichloromethane (DCM), then, $1 \mathrm{~mL}$ of DCM-dissolved $1.2 \mathrm{mg}$ a nearinfrared dye (DIR; Macklin) was added, poured into $13 \mathrm{~mL}$ of $3 \%$ polyvinyl alcohol solution, and emulsified in an ice bath at $45 \%$ power ultrasonication (JY92-IIN; Scientz) for $3 \mathrm{~min}$ to form an oilin-water emulsion. The emulsion was stirred overnight at room temperature to remove residual DCM. The nanoparticles were ultrafiltered through a $100 \mathrm{KD}$ ultrafiltration tube $(4,500 \mathrm{~g}, 10 \mathrm{~min}$; Millipore), and the underlying liquid was collected. The ultrafiltrate was fixed with pure water up to $6 \mathrm{~mL}$ and stored at $4^{\circ} \mathrm{C}$.

\section{Characterization of Nanoparticles}

The size and zeta potential of PLGA-TAS and MM-PLGA-TAS were determined at room temperature using a Malvern ZS90 nanoZetasizer. For testing stability, the nanoparticles were stored in $4^{\circ} \mathrm{C}$. PBS and the size and zeta potential of nanoparticles were measured at different time points. The morphology of PLGA-TAS and MM-PLGA-TAS nanoparticles was characterized by transmission electron microscopy (JEM-2100; JEOL). Ten milligrams of PLGA-TAS and MM-PLGA-TAS were dispersed in $10 \mathrm{~mL}$ of PBS ( $\mathrm{pH} 7.4$ ), and the samples were shaken at $200 \mathrm{rpm}$ at room 
temperature. The release of TAS from nanoparticles to PBS buffer at the indicated time points $(0,4,8,24,48$, and $72 \mathrm{~h})$ was determined by high-performance liquid chromatography (H-Class; Waters). The emission light spectra of MM-PLGA-TAS-DIR were measured using a microplate reader (VLB000GD0; Thermo Scientific, Waltham, MA, USA).

Preparation and Characterization of Membrane-Encapsulated Nanoparticles

The mouse macrophage cells (RAW264.7) with TLR4 overexpression were digested with ethylenediaminetetraacetic acid and resuspended in deionized water. The MMs were obtained according to the methods reported [17]. The mixture was sonicated several times, then $0.25 \mathrm{M}$ sucrose solution was immediately added and the membranes were collected by centrifugation. Cell membrane fragments were obtained by repeated freeze-thaw cycles. PLGA-TAS or PLGA-TAS/DIR were mixed with purified MMs (1:1 weight ratio of nanoparticles: membrane protein), and then the membrane-coated nanoparticles were obtained by extrusion (Avanti, Tonawanda, NY, USA) using a polycarbonate membrane with a pore size of $200 \mathrm{~nm}$. The particle size and morphology of the membrane-coated nanoparticles were analyzed by transmission electron microscopy. The particle size and zeta potential of the powders were further determined by the dynamic light scattering (DLS) method. For stability, the nanoparticles were stored in $4^{\circ} \mathrm{C}$ deionized water or PBS, and the size and zeta potential of the nanoparticles were determined at different time points.

\section{In vitro Cellular Uptake of MM-PLGA-TAS/DIR}

RAW264.7 cells were pretreated with LPS ( Sigma-Aldrich) and Tnfa recombinant protein (410-MT; R\&D Systems) for $12 \mathrm{~h}$, followed by coincubation with PLGA-TAS/DIR and MM-PLGATAS/DIR for $6 \mathrm{~h}$. Cells were then fixed with $4 \%$ paraformaldehyde for $15 \mathrm{~min}$, and nuclei were stained with 4-, 6-diamidino-2-phenylindole. Images were observed using a fluorescence microscope (BX53; Olympus).

\section{In vivo Biodistribution Evaluation}

For in vivo imaging, the BABL/C mice were treated with $4 \%$ DSS ( $36-50 \mathrm{kDa}$; MP Biomedicals) for 5 days, and then given oral PLGA-TAS/DIR or MM-PLGA-DIR (10 mg/kg); $12 \mathrm{~h}$ later an in vivo imaging system (IVIS) was used to measure the fluorescence intensity. The mice were then executed, and the colorectum was removed and placed in a $10 \mathrm{~cm}$ dish to detect the fluorescence intensity using IVIS. Fluorescence intensity was quantified with the Living Image software (PerkinElmer).

\section{Animals and Ulcerative Colitis Models}

Eight-week-old BABL/C female mice were purchased from Hunan SJA Laboratory Animal Co., Ltd. The mice were bred under specific pathogen-free conditions. All animal care and euthanasia protocols were approved by the Institutional Animal Care and Use Committee of Central South University (Changsha, China). Acute colitis was induced with $4 \%$ DSS for 7 consecutive days. The DSS solution was freshly prepared every other day. Mice were divided into 5 groups with 6 in each group: (1) negative control (i.e., no treatment), (2) only DSS treatment, (3) DSS + TAS (10 mg/kg; MedChemExpress), (4) DSS + PLGA-TAS (10 mg/kg TAS equivalent), and (5) DSS + MM-PLGA-TAS (10 mg/kg TAS equivalent).
The disease activity index (DAI) of the colitis model and H\&E staining for inflammation scoring methods were analyzed with reference to our published article [14].

Reverse Transcription and RT-qPCR

Colonic tissue total RNA was isolated using RNeasy Mini Kit (Qiagen), and cDNA was synthesized from $2 \mathrm{mg}$ total RNA with a Revert Aid RT Reverse Transcription Kit (Thermo Scientific). The RT-PCR analysis was performed using a Bio-Rad CFX96 RealTime System (Bio-Rad, Hercules, CA, USA). Mouse Gapdh was amplified in parallel as an internal control. Expression of each gene was quantified by measuring cycle threshold values, and the $2-$ DDCt method was used to calculate relative changes in gene expression. The primers for quantitative PCR were listed in online supplementary material. Table 1 ; for all online supplementary material, see www.karger.com/doi/10.1159/000519363.

\section{Immunohistochemistry}

Colonic paraffin-embedded sections were dewaxed and rehydrated. Immunohistochemical staining was performed to detect S100a9 (NB110-89726; Novus), Cd68 (clone KP1; Abcam), P65 (no. 4764; CST), and Stat3 (no. 9139; CST). Immunostained sections were observed microscopically and scored by percentage of positive cells and staining intensity: 0 : no staining, $1:<10 \%$ positive cells, $2: 11-50 \%$ positive cells, $3: 51-75 \%$ positive cells, $4:>75 \%$ positive cells; unstained $=0$, weakly stained $=1$, moderately stained $=2$, and strongly stained $=3$. The sum of the 2 scores was used as the expression intensity. Individual samples were evaluated by at least 2 pathologists in a blinded manner.

\section{Cell Culture}

Mouse macrophage cell RAW264.7 and colon cancer cell CT26 were purchased from the Cell Bank of the Chinese Academy of Sciences (Shanghai, China) and cultured in RPMI 1640 medium supplemented with $10 \%$ FBS. The full-length coding sequence of murine TLR4 was subcloned into the mammalian expression vector pcDNA3.1 $1^{+}$. TLR4 Plasmid transfection was performed by using Lipofectamine 3000 (Invitrogen), and TLR4 overexpression was confirmed by flow cytometry.

\section{Flow Cytometry}

For extracellular staining, RAW264.7 cells were incubated with anti-TLR4 (FAB2759P; R\&D Systems) antibody or an isotype control antibody combination at $4^{\circ} \mathrm{C}$ for $30 \mathrm{~min}$, followed by washing and analysis on a BD FACSCalibur.

\section{Apoptosis Detection}

A terminal deoxynucleotidyl transferase dUTP nick end labeling (TUNEL) assay was performed to detect apoptosis. TUNEL staining was performed using the 1-step TUNEL Apoptosis Detection Kit (C11026-2; Beyotime, Shanghai, China), and the procedure was performed according to the manufacturing instructions. The number of TUNEL-positive cells was captured by fluorescence microscopy, and the percentage of apoptotic cells was determined using Image J software.

\section{Immunofluorescence}

Cells were immersed in medium containing 3.7\% paraformaldehyde for $1 \mathrm{~h}$, then permeabilized with $0.5 \%$ Triton X-100, and blocked with normal goat serum. Anti-S100a9 antibody (26992-1-AP; Pro- 


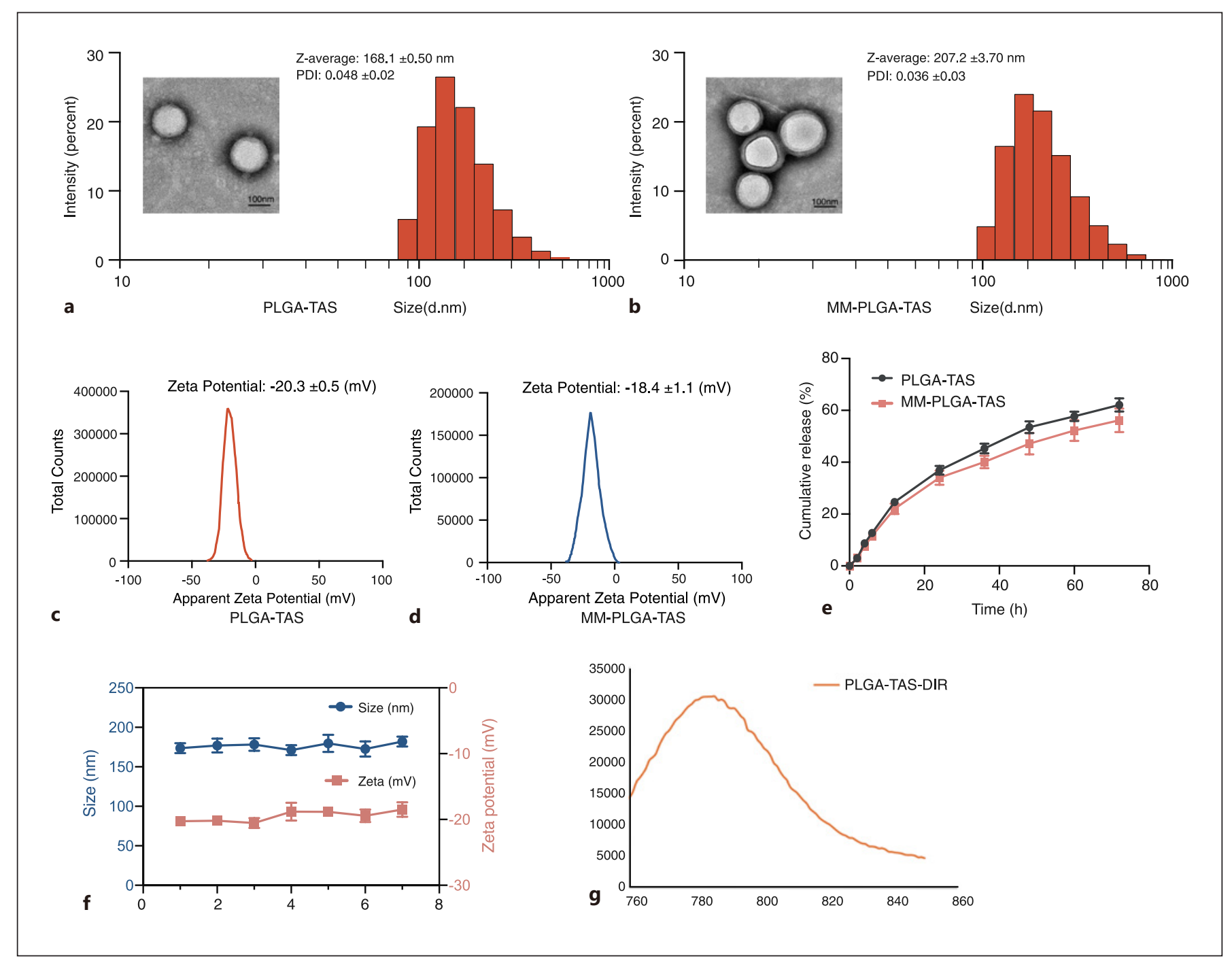

Fig. 1. Characterization of the TAS-loaded polymeric nanoparticles. The morphology of PLGA-TAS (a) and MM-PLGA-TAS (b) nanoparticles was characterized by transmission electron microscopy, and their size distribution was measured via DLS. Zeta potentials of PLGA-TAS (c) and MM-PLGA-TAS (d) nanoparticles. e Drug release kinetics from PLGA-TAS and MM-PLGA-TAS af-

teintech) was added and incubated for $12 \mathrm{~h}$ at $4^{\circ} \mathrm{C}$. Then, Alexa Fluor 488-conjugated antibody (Beyotime) was added and incubated for $1 \mathrm{~h}$. Images were observed using a fluorescence microscope.

\section{Cell Viability Assay}

The effect of nanoparticles on the viability of CT26 and RAW264.7 cells was analyzed using the MTT cell proliferation assay. Different concentrations of PLGA nanoparticles, MM-PLGA nanoparticles $(50,100,200$, and $500 \mu \mathrm{g} / \mathrm{mL})$ were added to the 96 well plates and incubated for $24 \mathrm{~h}$. Then, $20 \mu \mathrm{L}$ MTT solution (5 $\mathrm{mg} / \mathrm{mL}$; Beyotime) was added to each well and incubated for $4 \mathrm{~h}$. The absorbance was measured at $490 \mathrm{~nm}$ using a microplate reader.

Biomimetic Nanomaterials for Colitis Therapy ter different durations of dialysis. $\mathbf{f}$ Variations of average size and zeta potential of MM-PLGA-TAS in pH 7.4 PBS over 7 days. g Determination of MM-PLGA-TAS/DIR emission spectra by microplate marker. Data are shown as mean $\pm \mathrm{SD}(n=3)$. TAS, tasquinimod; PLGA, poly-lactic acid-glycolic acid; MM, macrophage membrane; DIR, infrared dye; DLS, dynamic light scattering.

\section{Statistical Analysis}

Statistical analyses were performed using SPSS18 and Prism 8.0. For normally distributed data, the significance of differences in means was determined using the 2-tailed paired or unpaired Student $t$ test; 2 -way ANOVA was used to compare data on mouse DAI and body weight changes between groups. Correlation coefficients were determined by Pearson's $r$ coefficient. $p$ values $<0.05$ were considered statistically significant. All data are incorporated into the article and its online supplementary material. 


\section{Results}

\section{Characterization of the Nanoparticles}

PLGA has many advantages, including biocompatibility, ease of preparation, slow-release action of drugs, and better stability compared with other types of drug delivery materials [16]. We used PLGA as a drug carrier and prepared MM-PLGA-TAS by using RAW264.7 cell membrane to coat PLGA-TAS nanoparticles. The average diameter of MM-PLGA-TAS nanoparticles was $207.2 \mathrm{~nm}$ and uncoated PLGA-TAS nanoparticles were approximately $168.1 \mathrm{~nm}$ as determined by DLS. Transmission electron microscopy images showed that PLGA-TAS and MM-PLGA-TAS are both spherical with average diameters of about $162 \mathrm{~nm}$ and $181 \mathrm{~nm}$, respectively, which are slightly smaller than the size observed by DLS, probably due to the hydration of nanoparticles in water in the DLS measurements (Fig. 1a, b). Macrophage cell membrane coating with a thickness of about $11 \mathrm{~nm}$ is observed by transmission electron microscopy. The drug loading and encapsulation efficiencies of TAS in PLGA were $6.37 \pm$ $0.26 \mu \mathrm{g} / \mathrm{mg}$ and $67.42 \pm 1.79 \%$, respectively, and the characteristics of other synthesized nanoparticles are shown in online supplementary material, Table 2 . The zeta potentials of PLGA-TAS and MM-PLGA-TAS after membrane coating were $-20.3 \pm 0.5 \mathrm{mV}$ and $-18.4 \pm 1.1 \mathrm{mV}$ (Fig. 1c, d). PLGA-TAS and MM-PLGA-TAS had polydispersity indices of 0.048 and 0.036 , respectively, indicating that it has excellent particle size distribution, which is consistent with the transmission electron microscopy observations. TAS was released in a time-dependent manner and MM-PLGA exhibited similar drug release even with membrane coating (Fig. 1e). Furthermore, these nanoparticles are stable under $4^{\circ} \mathrm{C}$ storage conditions, and their particle size and zeta potential (Fig. 1f) remain stable in deionized water for up to 1 week. The successful loading of DIR into nanoparticles was confirmed by using a microplate reader (Fig. 1f). These results indicate that the synthesized nanoparticles are suitable in terms of particle size and stability, with a good cell membrane coating effect, and suitable for conducting in vivo experiments in mice.

\section{The Ability of MM-PLGA-TAS to Target}

Inflammation in vitro and in vivo

To investigate the targeting ability of MM-PLGA-TAS to inflammatory regions, RAW264.7 cells were pretreated with LPS and TNFa for $12 \mathrm{~h}$. PLGA-TAS/DIR and MMPLGA-TAS/DIR were added to the cell culture supernatant and incubated for $6 \mathrm{~h}$, followed by observation of the fluorescence signal intensity. A fainter DIR fluorescence signal was observed in unactivated RAW264.7 cells and nanoparticles wrapped around MM showed lower macrophage uptake (Fig. 2a left). In contrast, a strong fluorescent signal was detected in RAW264.7 cells activated with LPS and TNFa (Fig. 2a right), indicating that macrophages with an inflammatory phenotype possess a stronger internalization of nanoparticles. Interestingly, the increased uptake of MM encapsulated nanoparticles was evident in macrophages of the inflammatory phenotype (Fig. 2a), which may be related to the increased cytokine secretion by macrophages in the inflammatory phenotype given the ability of macrophages to be chemotactic by the inflammatory environment [15]. S100s9 overexpression in inflammatory macrophages (online suppl. Fig. 2) may promote the ingestion of TLR4-overexpressing nanoparticles. In in vivo experiments, MM-PLGATAS/DIR had a significantly increased effect on colorectal regional targeting in colitis mice, but not in healthy mice (Fig. 2b). After $12 \mathrm{~h}$ of PLGA-TAS/DIR and MMPLGA-TAS/DIR administration, gastrointestinal tissues were collected from mice and IVIS imaging revealed that MM-PLGA-TAS/DIR was more enriched in the colorectal region (Fig. 2c). Fluorescence imaging of colitis tissues confirmed that oral administration of MM-PLGA-TAS/ DIR delivered nanoparticles efficiently into inflamed colonic tissues (Fig. 2d). Overexpression of TLR4 on the surface of MMs may be involved in this process, considering that the S100a9, a ligand of TLR4, is dramatically increased in inflammatory regions when inflammation occurs (Fig. 5b; online suppl. Fig. 1). These results suggest that nanoparticles encapsulating MMs could promote their enrichment within inflammatory regions and achieve better drug delivery.

\section{Oral Administration of MM-PLGA-TAS Ameliorated Acute Colitis in Mice}

We next investigated the therapeutic effect of nanoparticles on DSS-induced colitis in mice. The experimental procedure is shown in Figure 3a. Mice in the DSS group showed significant weight loss from day 2 and relatively high DAI levels compared with normal control mice (i.e., mice without DSS treatment) (Fig. 3b, c). In the DSS groups, nanoparticles (TAS, PLGA-TAS, and MMPLGA-TAS) treatment significantly improved weight loss and DAI scores. MM-PLGA-TAS was the most effective treatment for DSS mice, which is consistent with our previous results. The length of colon of MM-PLGA-TAStreated DSS mice was much longer than that of untreated DSS mice (Fig. 3d). 


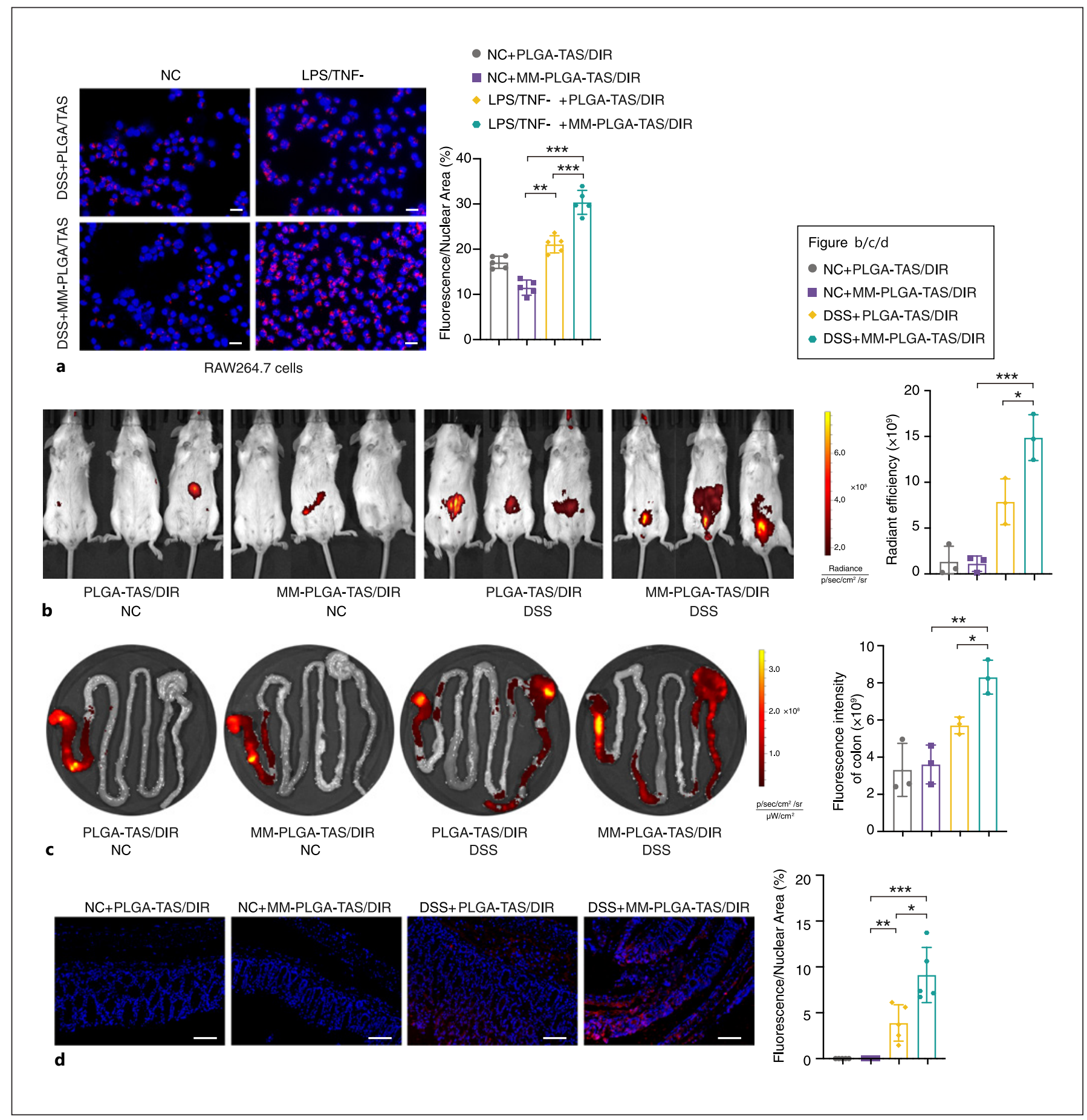

Fig. 2. Inflammation-targeting MM-PLGA-TAS in RAW264.7 cells and colitis mice. a RAW264.7 cells show increased effective uptake of nanoparticles in the inflammatory state. After treating RAW 264.7 cells with LPS and TNFa (or DMSO as negative controls) for $12 \mathrm{~h}$, cells were treated with PLGA-TAS/DIR or MMPLGA-TAS/DIR for $6 \mathrm{~h}$ and photographed using fluorescence microscopy. DAPI was used to label cell nuclei. Scale bars, $20 \mu \mathrm{m}$. PLGA-TAS/DIR or MM-PLGA-TAS/DIR were orally administrated to healthy controls and colitis mice for $12 \mathrm{~h}$, and then mice (b) or gastrointestinal tissues (c) images were obtained using an IVIS. d Representative fluorescent of normal mice and DSS-induced mice, which were treated with PLGA-TAS/DIR or MMPLGA-TAS/DIR. The percent of positive cells was measured. Scale bars, $100 \mu \mathrm{m}$. Two-tailed unpaired $t$ test. ${ }^{*} p<0.05,{ }^{* *} p<0.01,{ }^{* * *} p$ $<0.001$. NC, negative control; TAS, tasquinimod; PLGA, poly-lactic acid-glycolic acid; MM, macrophage membrane; DIR, infrared dye; DSS, dextran sulfate sodium; LPS, lipopolysaccharide; DAPI, 4-, 6-diamidino-2-phenylindole; IVIS, in vivo imaging system.
Biomimetic Nanomaterials for Colitis Therapy
J Innate Immun 2022;14:380-392 DOI: $10.1159 / 000519363$ 

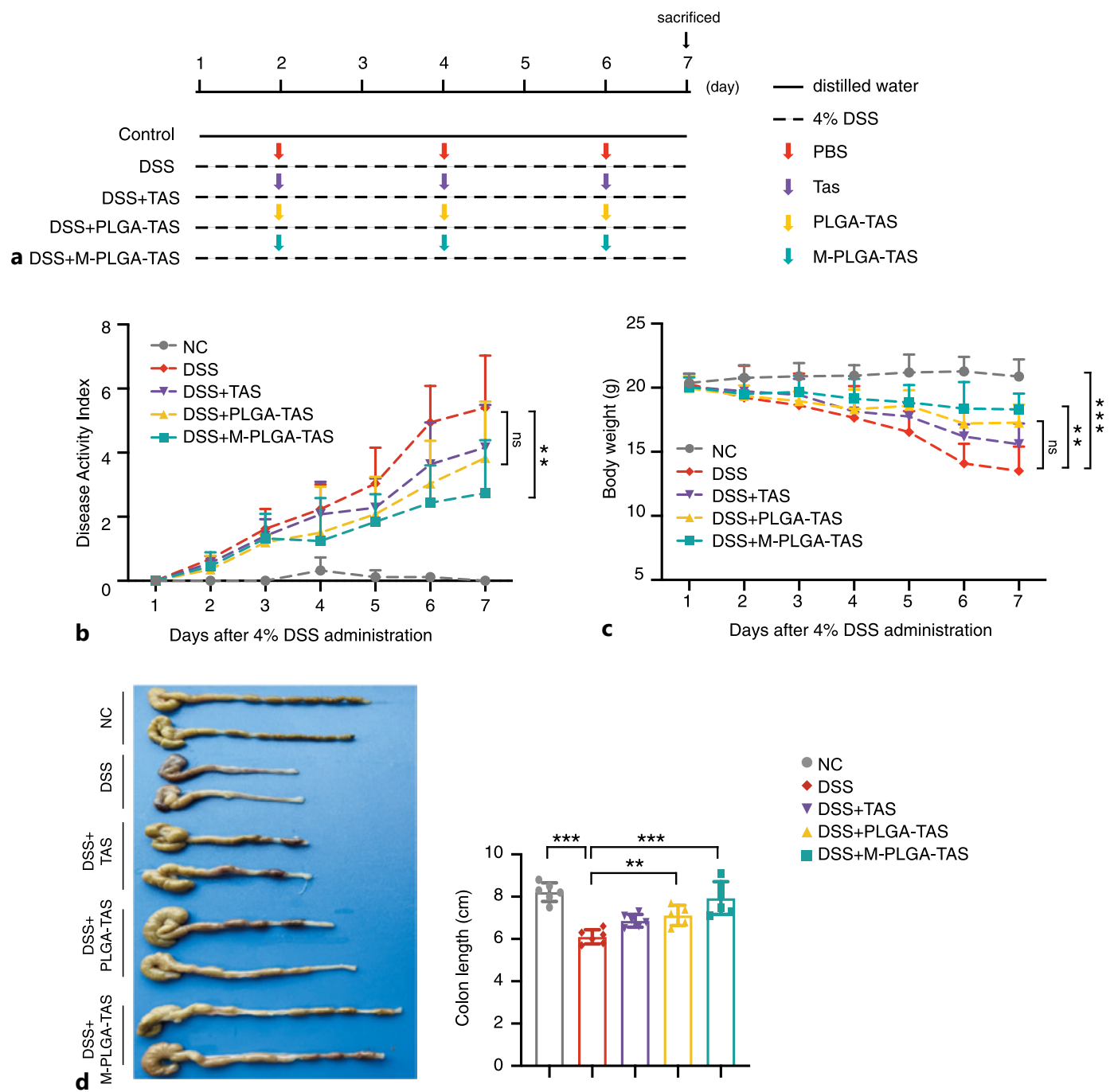

Fig. 3. Therapeutic effects of MM-PLGA-TAS treatment on DSSinduced acute colitis in mice. a BABL/C mice received either $4 \%$ DSS containing or distilled water alone (as NC) for 7 days. DSStreated mice are gavaged with TAS, PLGA-TAS, or MM-PLGATAS on days 1,3 , and $5 ; n=6$ per group. $\mathbf{b}$ The DAI was measured every day; DAI = weight loss score + stool characters score + hematochezia score. c Changes in body weight of mice during 7 days.

Histological analysis of colonic tissue from the DSS group mice showed severe inflammation, surface ulceration, crypt destruction, mucosal damage, and leukocyte infiltration (Fig. 4a, b). The severity of inflammation in the colonic tissues of mice from different treatment groups was scored, and both TAS and PLGA-TAS treatments alleviated the severity of inflammation, while the MM-PLGA-TAS treatment group had the least inflam- d The colon length was measured at day 7. Two-tailed unpaired $t$ test. ${ }^{* *} p<0.01,{ }^{* * *} p<0.001$. NC, negative control; TAS, tasquinimod; PLGA, poly-lactic acid-glycolic acid; MM, macrophage membrane; DSS, dextran sulfate sodium; LPS, lipopolysaccharide; DAPI, 4-, 6-diamidino-2-phenylindole; IVIS, in vivo imaging system; DAI, disease activity index.

matory response (Fig. 4b). DSS-induced colitis had toxic effects related to induction of apoptosis. Tissue TUNEL assays revealed that DSS was able to cause mouse colonic cell apoptosis, whereas MM-PLGA-TAS treatment alleviated the apoptosis (Fig. 4c). These results suggest that PLGA-TAS and MM-PLGA-TAS are effective in the treatment of DSS-induced acute colitis. 


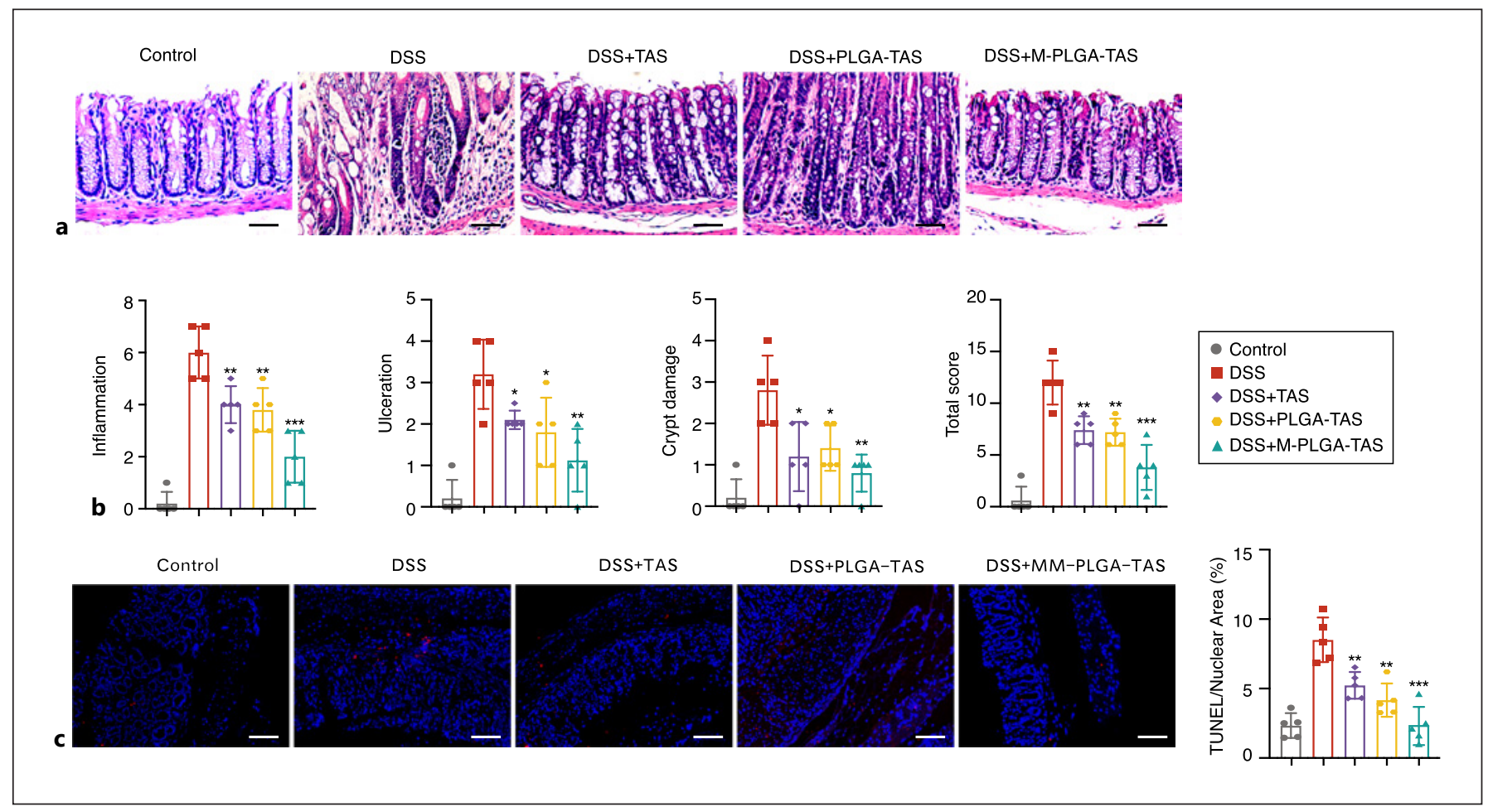

Fig. 4. MM-PLGA-TAS ameliorates inflammatory response of DSS-induced colitis in mice. a Representative H\&E-stained colon sections are shown. Scale bars, $50 \mu \mathrm{m}$. b Colon inflammation, ulceration, and crypt damage were scored individually, and composite total score was scored. $n=5$ per group. $\mathbf{c}$ Representative TUNEL staining of normal mice and DSS-induced mice, which were treated with PLGA-TAS or MM-PLGA-TAS. The percent of positive

To better understand the mechanisms by which PLGATAS and MM-PLGA-TAS improve symptoms in colitis mice, we assessed the mRNA expression levels of key cytokines in mice. Expression levels of S100a9, IL-6, Tnfo, $I L 1 \beta$, and iNos were significantly increased in the DSS mice, and these pro-inflammatory molecules may have exacerbated the extent of ulcerative colitis in DSS mice (Fig. 5a). The expression levels of S100a9 in DSS mice were significantly increased and may be critical for the recruiting of MM-PLGA-TAS nanoparticles to inflammatory regions. Most cytokines were decreased in the TAS, PLGA-TAS, and MM-PLGA-TAS-treated groups compared to the DSS group, except for IL-10, which has profound anti-inflammatory functions (Fig. 5a). Immunohistochemistry assay observed that $\mathrm{Cd}^{+} 8^{+}$positive macrophages were recruited to colonic tissues in the DSS mice and were decreased in the nanoparticle-treated group (Fig. 5b). Considering the results from Figure 2a, macrophages may play a role in promoting the uptake of cells was measured. Five fields were counted per mouse. Scale bars, $100 \mu \mathrm{m}$. Two-tailed unpaired $t$ test. ${ }^{*} p<0.05,{ }^{* *} p<0.01,{ }^{* * *} p<$ 0.001 compared with DSS group. NC, negative control. TAS, tasquinimod; PLGA, poly-lactic acid-glycolic acid; MM, macrophage membrane; DSS, dextran sulfate sodium; TUNEL, terminal deoxynucleotidyl transferase dUTP nick end labeling.

MM-PLGA-TAS nanoparticles in the colon, and more macrophage infiltration could aid in the rapid uptake and internalization of nanoparticles in the tissues. NF- $\kappa \mathrm{B}$ and Stat3 pathways play a key role in the development of colitis in DSS mice [18], we observed that the expression levels of P65 and Stat 3 molecules were downregulated in colonic tissues of TAS and nanoparticle-treated mice, which is consistent with the observed reduction in tissue inflammation (Fig. 5b). In conclusion, MM-PLGA-TAS was able to enrich heavily into the inflammatory regions and inhibit the activity of S100a9 by releasing TAS drugs. TLR4 on the surface of MMs may be involved in the process of adsorption of excess S100a9 molecules; and the expression of P65 and Stat 3 molecules was reduced, thus achieving relief of colorectal inflammation in mice. These results suggest that MM-PLGA-TAS is a promising oral nano-delivery drug system for treatment of ulcerative colitis. 

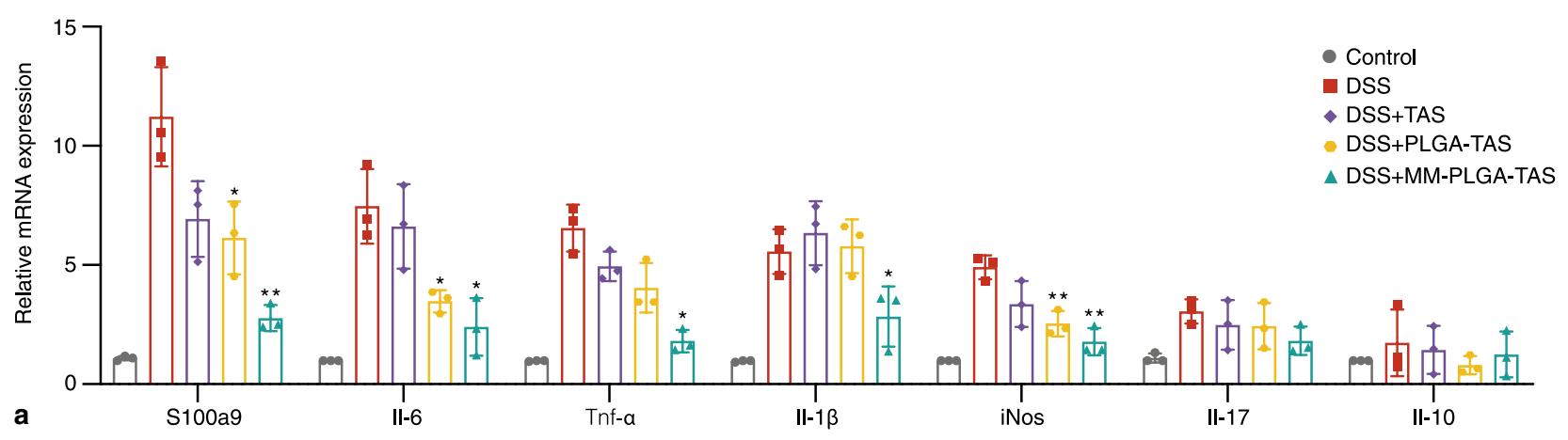

NC

DSS

DSS+TAS

DSS+PLGA-TAS

DSS+MM-PLGA-TAS
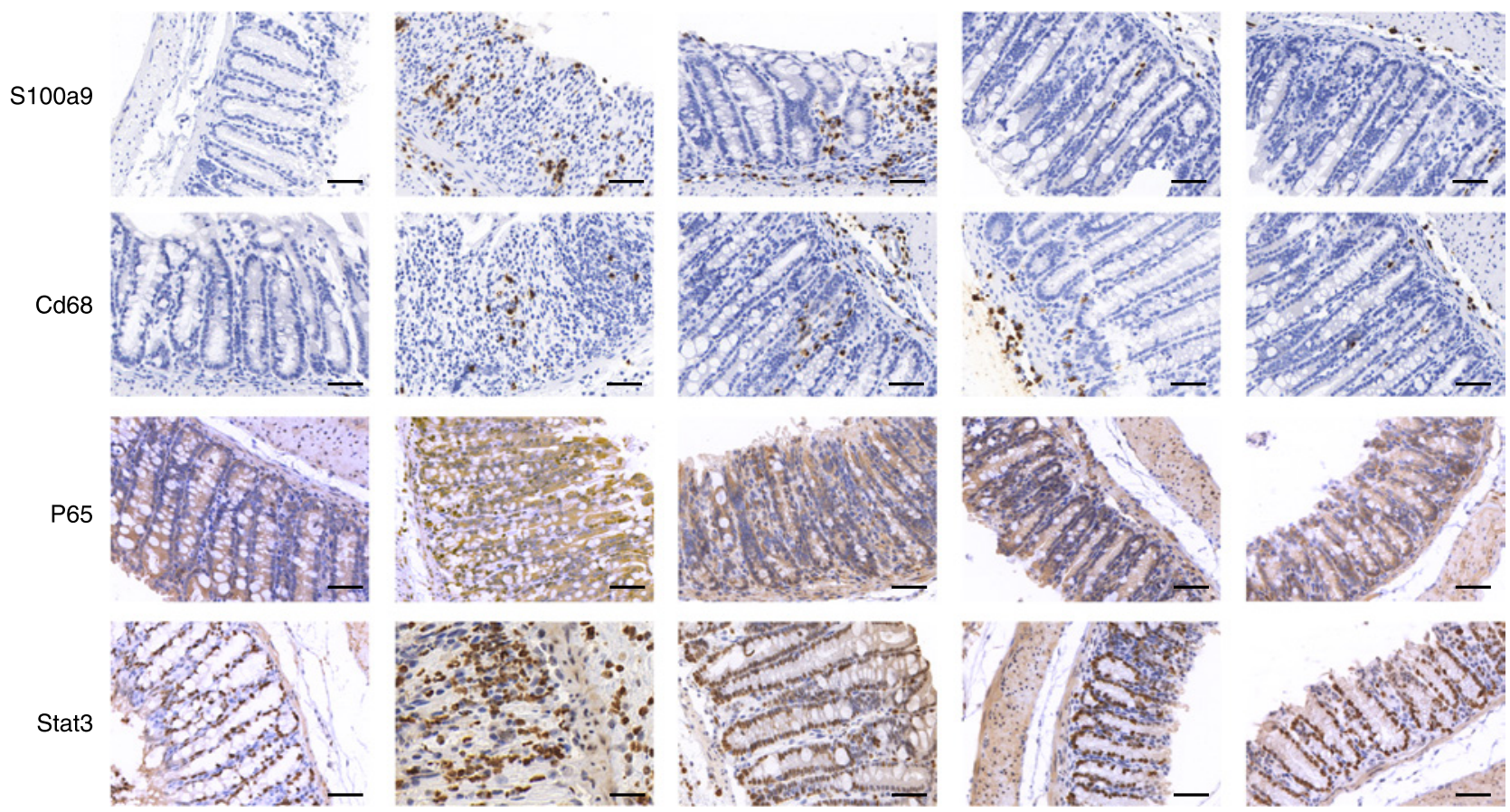

- NC

DSS
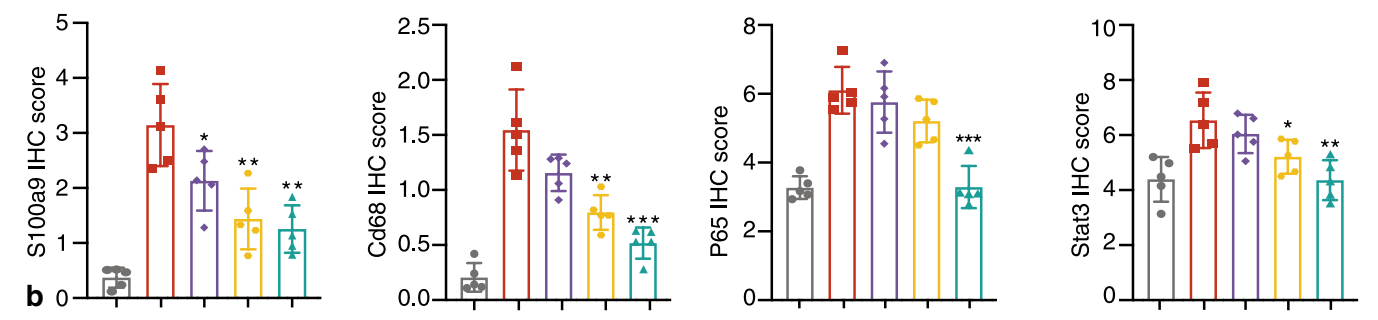

DSS+TAS

- DSS+PLGA-TAS

$\triangle$ DSS+MM-PLGA-TAS

Fig. 5. Expression levels of key molecules of inflammatory factors and signaling pathways in the colorectum of DSS-induced colitis mice. a Expression of S100a9, Il-6, Tnfo, Il1 $\beta$, iNos, Il17, and Il10 mRNA, as assessed by quantitative real-time PCR in normal control and TAS, PLGA-TAS, or MM-PLGA-TAS treated colitis tissues. b Expression of S100a9, Cd68, P65 and Stat3 proteins, as as- sessed by IHC in normal control and TAS, PLGA-TAS or MMPLGA-TAS treated colitis tissues. Scale bars, $50 \mu \mathrm{m}$. Two-tailed unpaired $t$ test. ${ }^{*} p<0.05,{ }^{* *} p<0.01,{ }^{* * *} p<0.001$ compared with DSS group. NC, negative control; TAS, tasquinimod; PLGA, polylactic acid-glycolic acid; MM, macrophage membrane; DSS, dextran sulfate sodium; IHC, immunohistochemistry. 


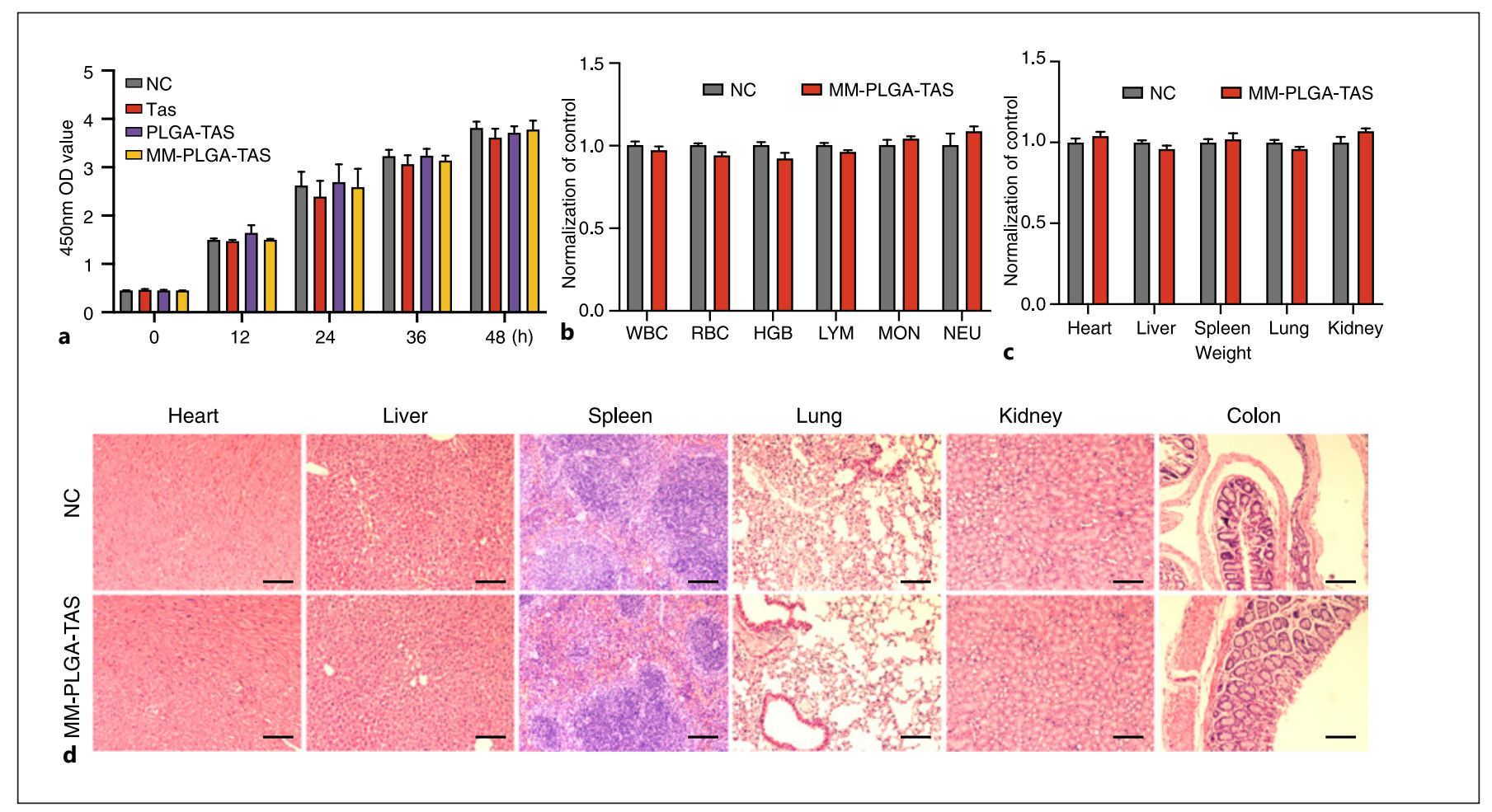

Fig. 6. Preliminary biosafety evaluation of nanoparticles. a CT26 cells were treated with TAS, PLGA-TAS or MM-PLGA-TAS, and the absorbance at $450 \mathrm{~nm}$ at different time points was measured using an enzyme labeling plate after the addition of CCK8. b Normal BABL/C mice were treated with TAS, PLGA-TAS, or MMPLGA-TAS and then subjected to hematological analysis. $c$ Weight of heart, liver, spleen, lung, and kidney in control and MM-PLGATAS-treated mice. There was no significant difference among the

Biocompatibility of MM-PLGA-TAS in vitro and in vivo The biocompatibility of nanomaterials remains a key issue that requires careful consideration because of the presence of a complicated immune system in mice. To analyze the potential cytotoxicity, we investigated the effects of TAS, PLGA-TAS, and MM-PLGA-TAS on mouse colon cancer cell CT26 in vitro. TAS, PLGA-TAS, and MMPLGA-TAS were added to the supernatant of CT26 cell culture, respectively, and treated the CT26 cells for indicated time $(0,12,24,36$, and $48 \mathrm{~h})$. The growth rate of CT26 cells had not been affected by the different in vitro treatments (Fig. 6a). In addition, nanoparticles (PLGA and MM-PLGA) treatment with different doses had no significant effect on growth rate of CT26 and RAW264.7 cells (online suppl. Fig. 3). To study the in vivo toxicity of nanoparticles, BABL/C mice were orally treated with MM-PLGA-TAS or PBS for 7 days, followed by blood collection for hematological analysis (Fig. 6b). Gastrointesti- groups. d. Major organs for H\&E staining. There was no significant difference among the groups. Scale bars, $100 \mu \mathrm{m}$. There were no pathological changes. Data are shown as mean $\pm \mathrm{SD}(n=3)$. TAS, tasquinimod; PLGA, poly-lactic acid-glycolic acid; MM, macrophage membrane; DSS, dextran sulfate sodium; IHC, immunohistochemistry; WBCs, white blood cells; RBCs, red blood cells; HGB, hemoglobin; LYMs, lymphocytes; MONs, monocytes and NEUs, neutrophils. nal tract tissues and major organ (heart, liver, spleen, lung, and kidney) tissues were collected for histological analysis. There were no significant differences in organ weight parameters in MM-PLGA-TAS-treated mice compared to negative controls (Fig. 6c). No signs of inflammation or histological alterations were found in the heart, liver, spleen, lung, or kidney tissues of MM-PLGA-TAS-treated mice (Fig. 6d). These results suggest that MM-PLGA-TAS is not toxic in vivo to healthy mice. In conclusion, MMPLGA-TAS has good biocompatibility in vitro and in vivo and can be used as a safe drug delivery system in mice.

\section{Discussion}

Development of safe and effective targeted colonic drug delivery systems is an urgent need for the treatment of ulcerative colitis. Nano-delivery systems with tissue- 
specific targeted drug delivery have been rapidly developed as a novel and effective tool for the treatment of ulcerative colitis. Oral drug delivery is an effective route for nanomaterials in the treatment of ulcerative colitis due to the ability of nanomaterials to protect the drug through the harsh gastrointestinal environment and reach the desired targeting area [19]. Unlike systemic administration by intravenous injection, orally targeted drugs allow direct drug enrichment to the area where colorectal inflammation occurs, reducing systemic adverse drug reactions and increasing local tissue drug concentrations, which is expected to accelerate the recovery of inflammation. Our results show that MM-encapsulated PLGA nanocarriers are better enriched to inflammatory regions than conventional PLGA direct oral administration, which is consistent with our previous finding of macrophage recruitment by S100A9 in inflammatory regions [13].

An important drawback with PLGA nanoparticles is the limited types of functional groups available on the surface for conjugation to targeting ligands, which limit the function of PLGA materials [20]. Progress has been made in targeting inflammation with cell membrane-encapsulated bionanomaterials. For example, polymeric nanoparticles synthesized using neutrophil membrane coating inherit the antigenic outer membrane function and associated membrane function of the source cells, giving them the ability to target inflammatory regions of joints [21]. Compared to synthetic materials, MMs have the best biocompatibility and are able to evade nonspecific uptake by macrophages, thereby increasing the residence time of the material in the tissue. The surface of MMs is rich in inflammation-related receptors, including TLR family, IL6 receptor family, etc. These unique inflammation molecule-related receptors make MMs known as better inflammation-targeting nanocoatings. In the current study, we chose to utilize natural macrophage cell membranes to overcome the limitations of PLGA. Nanoparticles wrapping with MMs reduced their endocytosis by macrophages, had longer tissue retention time, and were more aggregated in the inflammatory tissue regions. In addition, S100A9 can recruit a variety of immune cells, such as neutrophils, dendritic cells, and monocytes, which together orchestrate the disease process in ulcerative colitis mice [14]. The membranes of these immune cells can likewise be used as nanocoating for targeted treatment of ulcerative colitis when combined with other nanotherapeutic agents.

Inflammatory mediator S100A9 is closely associated with ulcerative colitis $[14,22,23]$, and we previously developed an anti-S100a9 antibody to successfully treat a mouse model of ulcerative colitis. However, antibodies bind to S100A9 in the blood during intravenous administration, reducing the accumulation of antibodies in the area of inflammation. Therefore, we considered the development of drug delivery systems that specifically target inflammation. S100A9 is able to bind to TLR4 on the surface of macrophages and activate downstream Nf- $\kappa b$ and Stat3 signaling pathways [24, 25]. Tasquinimod, a quinoline-3-carboxamide analog, has immunomodulatory effects and is an inhibitor of S100a9 [26]. Nanomaterial cell membrane overexpression of TLR4 enriches the material to inflammatory regions via ligand-receptor binding and is able to neutralize excess S100a9 in inflammatory regions, synergizing with S100a9 inhibitors. Mice with colitis treated with MM-PLGA-TAS produced fewer inflammatory factors, such as Tnf $\alpha, I L 1 \beta, I L 6, I L 17 a$, and IL12a. Colitis-associated cancer occurs in $>20 \%$ of patients within 30 years of disease onset during the recurrent progression of IBD [27]. Our previous analysis of the dynamic process of AOM/DSS-induced colorectal inflammation-cancer transformation revealed abnormal activation of some signaling pathways (e.g., Nf-kb, Stat3, and MAPK signalings) during the inflammatory phase, suggesting these signalings play a decisive role in inflammation-cancer link [18]. MM-PLGA-TAS treatment decreased the expression of key molecules of these inflammatory pathways, implying that MM-PLGA-TAS can alleviate the symptoms of enteritis in ulcerative colitis mice by decreasing the expression of inflammatory signaling pathways and inflammatory factors. In addition, MMPLGA-TAS reduced DSS-induced cell death and maintained regenerative proliferation of intestinal epithelial cells, indicating that MM-PLGA-TAS has a protective effect on the intestinal mucosal barrier. Compared with systemic administration, PLGA-TAS was able to obtain higher drug concentrations in tissues while reducing the systemic toxicity of the drug. MM-PLGA-TAS further enhanced the targeting of nanoparticles for colitis tissues, while MMs were able to neutralize inflammatory factors, such as S100a9, a unique property that is the key to improving the therapeutic effect of nanoparticles on colitis.

In summary, we developed a convenient oral delivery system to specifically increase the aggregation of nanoparticles at sites of intestinal inflammation in mice by coating the membranes of macrophages overexpressing TLR4 and exploiting their inflammatory homing effect. Local inflammation was regulated and suppressed by the adsorption of multiple inflammatory factors through abundant receptors on the surface of MMs. The MM-PLGATAS system significantly attenuated the symptoms of 
DSS-induced colitis in mice and reduced the expression of inflammation signaling pathways and inflammatory factors. This system may be a promising approach for the treatment of ulcerative colitis.

\section{Acknowledgments}

We thank Zhanwei Zhu (Xiangya Hospital, Central South University) for providing IVIS technical assistance; and Nanjing XFNANO Materials Tech Co., Ltd for technical support of nano synthesis.

\section{Statement of Ethics}

All animal care and euthanasia protocols were approved by the Institutional Animal Care and Use Committee of Central South University (Changsha, China).

\section{Conflict of Interest Statement}

The authors declare no conflict of interest.

\section{Funding Sources}

This work was funded by the National Natural Science Foundation of China (81874170, 82073261), and China 111 Project (1112-12), Independent Exploration and Innovation Project of Central South University (2020zzts226, 2020zzts769, 206501024, 196511077), Natural ScienceFoundation ofChangsha(kq2014297), and Hunan Province Natural Sciences Foundation of China (2021JJ31116).

\section{Author Contributions}

Z.L. and J.M. designed and conceived the experiments. Z.L., X.Z., C.L., Q.P., Y.W., R.Z., and Q.Y. performed the experiments and analyzed the data. L.Z. and J.M. wrote the manuscript.

\section{Data Availability Statement}

All data generated or analyzed during this study are included in this article and its online supplementary material files. Further inquiries can be directed to the corresponding author.

\section{References}

1 Kozuch PL, Hanauer SB. Treatment of inflammatory bowel disease: a review of medical therapy. World J Gastroenterol. 2008 Jan 21;14(3):354-77.

2 Torres J, Billioud V, Sachar DB, Peyrin-Biroulet $\mathrm{L}$, Colombel JF. Ulcerative colitis as a progressive disease: the forgotten evidence. Inflamm Bowel Dis. 2012 Jul;18(7):1356-63.

3 Hoivik ML, Moum B, Solberg IC, Henriksen $\mathrm{M}$, Cvancarova M, Bernklev T, et al. Work disability in inflammatory bowel disease patients 10 years after disease onset: results from the IBSEN Study. Gut. 2013 Mar;62(3):368-75.

4 Bernstein CN. Treatment of IBD: where we are and where we are going. Am J Gastroenterol. 2015 Jan;110(1):114-26.

5 Neurath MF. Current and emerging therapeutic targets for IBD. Nat Rev Gastroenterol Hepatol. 2017 May;14(5):269-78.

6 Schreiber S, Colombel JF, Bloomfield R, Nikolaus S, Schölmerich J, Panés J, et al. Increased response and remission rates in shortduration Crohn's disease with subcutaneous certolizumab pegol: an analysis of PRECiSE 2 randomized maintenance trial data. Am J Gastroenterol. 2010 Jul;105(7):1574-82.

7 Peyrin-Biroulet L, Lémann M. Review article: remission rates achievable by current therapies for inflammatory bowel disease. Aliment Pharmacol Ther. 2011 Apr;33(8):870-9.

8 Wilson DS, Dalmasso G, Wang L, Sitaraman SV, Merlin D, Murthy N. Orally delivered thioketal nanoparticles loaded with TNF- $\alpha$ -
siRNA target inflammation and inhibit gene expression in the intestines. Nat Mater. 2010 Nov;9(11):923-8.

9 Llewellyn SR, Britton GJ, Contijoch EJ, Vennaro $\mathrm{OH}$, Mortha $\mathrm{A}$, Colombel JF, et al. Interactions between diet and the intestinal microbiota alter intestinal permeability and colitis severity in mice. Gastroenterology. 2018 Mar; 154(4):1037-46.e2.

10 Li R, He Y, Zhu Y, Jiang L, Zhang S, Qin J, et al. Route to rheumatoid arthritis by macrophage-derived microvesicle-coated nanoparticles. Nano Lett. 2019 Jan 9;19(1):124-34.

11 Gao C, Huang Q, Liu C, Kwong CHT, Yue L, Wan JB, et al. Treatment of atherosclerosis by macrophage-biomimetic nanoparticles via targeted pharmacotherapy and sequestration of proinflammatory cytokines. Nat Commun. 2020 May 26;11(1):2622.

12 Wang S, Song R, Wang Z, Jing Z, Wang S, Ma J. S100A8/A9 in inflammation. Front Immunol. 2018;9:1298.

13 Zhang X, Ai F, Li X, She X, Li N, Tang A, et al. Inflammation-induced S100A8 activates Id 3 and promotes colorectal tumorigenesis. Int J Cancer. 2015 Dec 15;137(12):2803-14.

14 Zhang X, Wei L, Wang J, Qin Z, Wang J, $\mathrm{Lu} \mathrm{Y}$, et al. Suppression colitis and colitis-associated colon cancer by anti-S100a9 antibody in mice. Front Immunol. 2017;8:1774.

15 Li Z, Wang J, Zhang X, Liu P, Zhang X, Wang $\mathrm{J}$, et al. Proinflammatory S100A8 induces PDL1 expression in macrophages, mediating tu- mor immune escape. J Immunol. 2020 May 1; 204(9):2589-99.

$16 \mathrm{Mu} \mathrm{L}$, Feng SS. A novel controlled release formulation for the anticancer drug paclitaxel (Taxol): PLGA nanoparticles containing vitamin E TPGS. J Control Release. 2003 Jan 9; 86(1):33-48.

17 Liu L, Wang Y, Guo X, Zhao J, Zhou S. A biomimetic polymer magnetic nanocarrier polarizing tumor-associated macrophages for potentiating immunotherapy. Small. 2020 Sep;16(38):e2003543.

18 Tang A, Li N, Li X, Yang H, Wang W, Zhang $\mathrm{L}$, et al. Dynamic activation of the key pathways: linking colitis to colorectal cancer in a mouse model. Carcinogenesis. 2012 Jul;33(7): 1375-83.

19 Zhang M, Merlin D. Nanoparticle-based oral drug delivery systems targeting the colon for treatment of ulcerative colitis. Inflamm Bowel Dis. 2018 Jun 8;24(7):1401-15.

20 Toti US, Guru BR, Grill AE, Panyam J. Interfacial activity assisted surface functionalization: a novel approach to incorporate maleimide functional groups and cRGD peptide on polymeric nanoparticles for targeted drug delivery. Mol Pharm. 2010 Aug 2;7(4):1108-17.

21 Zhang Q, Dehaini D, Zhang Y, Zhou J, Chen $X$, Zhang L, et al. Neutrophil membrane-coated nanoparticles inhibit synovial inflammation and alleviate joint damage in inflammatory arthritis. Nat Nanotechnol. 2018 Dec; 13(12):1182-90. 
22 Lee MJ, Lee JK, Choi JW, Lee CS, Sim JH, Cho $\mathrm{CH}$, et al. Interleukin-6 induces S100A9 expression in colonic epithelial cells through STAT3 activation in experimental ulcerative colitis. PLoS One. 2012;7(9):e38801.

23 Su S, Kong W, Zhang J, Wang X, Guo H. Integrated analysis of DNA methylation and gene expression profiles identified S100A9 as a potential biomarker in ulcerative colitis. Biosci Rep. 2020 Dec 23;40(12):40.
24 Sunahori K, Yamamura M, Yamana J, Takasugi $\mathrm{K}$, Kawashima M, Yamamoto $\mathrm{H}$, et al. The S100A8/A9 heterodimer amplifies proinflammatory cytokine production by macrophages via activation of nuclear factor kappa $\mathrm{B}$ and p38 mitogen-activated protein kinase in rheumatoid arthritis. Arthritis Res Ther. 2006;8(3):R69.

25 Vogl T, Tenbrock K, Ludwig S, Leukert N, Ehrhardt C, van Zoelen MA, et al. Mrp8 and Mrp14 are endogenous activators of Toll-like receptor 4, promoting lethal, endotoxin-induced shock. Nat Med. 2007 Sep;13(9):10429.
26 White JR, Phillips F, Monaghan T, Fateen W, Samuel S, Ghosh S, et al. Review article: novel oral-targeted therapies in inflammatory bowel disease. Aliment Pharmacol Ther. 2018 Jun; 47(12):1610-22.

27 Lakatos PL, Lakatos L. Risk for colorectal cancer in ulcerative colitis: changes, causes and management strategies. World J Gastroenterol. 2008 Jul 7;14(25):3937-47. 